\title{
Archivos

\section{Papel de la inflamación renal en la fisiopatología de la hipertensión sensible a sal}

\author{
Yaniel Castro Torres ${ }^{a, *}$, Alejandro Emilio Santos Portela ${ }^{a}$ e Ildiko María Garrido Bősze ${ }^{b}$
}

a Facultad de Medicina, Universidad de Ciencias Médicas Dr. Serafín Ruiz de Zárate Ruiz, Santa Clara, Villa Clara, Cuba

b Policlínico Docente Universitario José Ramón León Acosta, Santa Clara, Villa Clara, Cuba

Recibido el 7 de agosto de 2013; aceptado el 10 de febrero de 2014

\author{
PALABRAS CLAVE \\ Hipertensión sensible \\ a sal; \\ Estrés oxidativo; \\ Inflamación renal; \\ Linfocitos T; \\ Macrófagos; \\ Cuba
}

\section{KEYWORDS}

Salt-sensitive

hypertension;

Oxidative stress;

Renal inflammation;

T-lymphocytes;

Macrophages;

Cuba
Resumen La hipertensión sensible a sal es el aumento de la presión arterial luego de una sobrecarga salina, como consecuencia esencialmente de una disminución en la excreción renal de sodio. En los últimos años, ha sido desarrollada una teoría para explicar su origen que tiene como base la inflamación del tejido renal. El proceso inicia con la producción en los riñones de radicales libres derivados del metabolismo oxidativo. Luego se desarrolla un mecanismo de inflamación del intersticio renal por infiltración de linfocitos T, y otras células inmunológicas. Fundamentalmente los linfocitos $\mathrm{T}$ incrementan la producción de angiotensina ॥ que estimula la retención de sodio y agua a este nivel, favoreciendo el desarrollo de hipertensión sensible a sal. La relación entre infiltración renal por células del sistema inmune e hipertensión sensible a sal permite, en parte, explicar la asociación entre enfermedades autoinmunes y la hipertensión arterial. El uso de antioxidantes y el diseño de nuevos fármacos pueden ser una alternativa adicional al tratamiento de los pacientes afectados.

( 2013 Instituto Nacional de Cardiología Ignacio Chávez. Publicado por Masson Doyma México S.A. Todos los derechos reservados.

Role of renal inflammation in the physiopathology of salt-sensitive hypertension

Abstract Salt-sensitive hypertension is produced by a decrease in salt renal excretion after a salt overload. Over the last few years, a new theory has been developed to explain this condition based on renal tissue inflammation. This process begins with free radicals production in renal tissue due to oxidative metabolism. Then they favor a renal inflammation mechanism with T-lymphocytes infiltration and other immune cells. Essentially, T-lymphocytes determine an increase in angiotensin $॥$ production which raises sodium and water retention. Association among autoimmune diseases and hypertension may be explained, in part, by the relationship between salt-sensitive hypertension and renal inflammation. The use of antioxidant drugs and

\footnotetext{
* Autor para correspondencia: Universidad de Ciencias Médicas «Dr. Serafín Ruiz de Zárate Ruiz», Luz Caballero \#161 entre Hospital y Alejandro Oms, Reparto Parroquia. Santa Clara, CP 50100 Villa Clara, Cuba, Teléfono: +53 42272776.

Correos electrónicos: yanielct@edu.vcl.sld.cu, castrotorresy@gmail.com (Y. Castro Torres).
} 
the development of new medicaments may be a choice for treating patients affected with this condition

(c) 2013 Instituto Nacional de Cardiología Ignacio Chávez. Published by Masson Doyma México S.A. All rights reserved.

\section{Introducción}

Actualmente la hipertensión arterial (HTA) representa un problema de salud para gran parte de la población mundial. Se estima que afecta al $25-47 \%$ de las personas mayores de 18 años $^{1}$, y provoca alrededor de 7.6 millones de muertes prematuras anualmente ${ }^{2}$. El aumento de las cifras de presión arterial (PA) por encima de los valores establecidos favorece el incremento en la incidencia de infarto agudo de miocardio, accidentes vasculares encefálicos, insuficiencia renal, muerte súbita y enfermedad vascular periférica ${ }^{3}$.

La ingestión excesiva de sal constituye un factor de riesgo para el desarrollo de HTA esencial ${ }^{4,5}$. En países donde el consumo de esta sustancia se encuentra entre los 100 y los $500 \mathrm{mEq} /$ día, la incidencia de HTA es mayor, cuando se compara con los que mantienen una dieta inferior a $50 \mathrm{mEq} / \mathrm{di}^{6}{ }^{6}$. Los mecanismos involucrados incluyen el incremento en el volumen intravascular y la resistencia vascular periférica, debido en parte a la activación del sistema nervioso simpático ${ }^{7}$.

La hipertensión sensible a sal (HSS) es el aumento de la PA luego de una sobrecarga salina, y tiene una frecuencia cercana al $80 \%$ en pacientes mayores de 60 años $^{8}$. Muchas han sido las propuestas para explicar la deficiencia en la excreción urinaria de sal en individuos que desarrollan esta condición ${ }^{9}$, sugiriéndose la acción de factores vasoactivos y natriuréticos como el óxido nítrico, el factor natriurético auricular y el sistema cininas-kalikreína ${ }^{10}$. En los últimos años varios investigadores han centrado su atención en la relación entre el estrés oxidativo, la infiltración del intersticio renal por células inflamatorias y el desarrollo de HSS. Esto permite plantear nuevas hipótesis que explican la relación entre HTA y enfermedades autoinmunes teniendo como base los mecanismos de la inflamación renal.

La presente revisión se propuso mostrar las evidencias que apoyan la relación entre la formación de radicales libres $(\mathrm{RL})$ de oxígeno y nitrógeno, la inflamación del intersticio renal por acción de células del sistema inmunológico y la subsiguiente producción de hormonas prohipertensivas que explican el deterioro de la excreción renal de sodio en pacientes con HSS. Igualmente se analizarán algunos aspectos en el orden práctico sobre la base de estos conocimientos, que pueden ser útiles en el propósito de alcanzar una reducción en las cifras tensionales en la población tanto hipertensa como no hipertensa.

\section{Hipertensión sensible a sal: concepto y epidemiología}

La HSS es el aumento de la PA media mayor de $10 \mathrm{mmHg}$ luego de ingerir una dieta rica en sodio, seguido de un contexto donde existe un aumento en la depleción de este catión debido al uso de diuréticos y/o dietas hiposódicas ${ }^{11}$. Esta condición al parecer tiene una mayor prevalencia entre individuos en edades avanzadas de la vida. Aproximadamente el $80 \%$ de las personas hipertensas mayores de 60 años padecen HSS, mientras que entre los menores de 40 años solo la manifiestan el $50 \%{ }^{9}$. Ciertos elementos epidemiológicos se han asociado con mayor frecuencia a esta condición, como la raza negra, obesidad, diabetes mellitus, disfunción renal, uso de ciclosporina $A$ y la edad avanzada ${ }^{12}$. Además existen evidencias de trastornos en la expresión de genes que codifican para el angiotensinógeno ${ }^{13}$, la enzima convertidora de angiotensina $^{14}$, la renina ${ }^{15}$ y la sintetasa de aldosterona ${ }^{16}$. Como vemos todos son elementos que integran el sistema renina-angiotensina-aldosterona que desempeñan un papel fundamental en la fisiopatología de la HSS.

Se ha observado que los pacientes con HSS tienen un riesgo incrementado de sufrir daños en órganos diana, pues padecen con mayor frecuencia de hipertrofia ventricular izquierda, proteinuria y bloqueo del descenso nocturno de la $\mathrm{PA}^{17}$. Estos efectos negativos incrementan la probabilidad de eventos cardiovasculares entre hipertensos con sensibilidad a la sal, en comparación con los resistentes ${ }^{18}$.

\section{El estrés oxidativo y su relación con la hipertensión arterial}

El conocimiento de los RL y su metabolismo ha permitido descifrar un grupo de funciones que desarrollan estas sustancias en el organismo. Los mismos están integrados a la regulación del tono vascular, la transducción de señales y como sensores de la tensión de oxígeno en los tejidos ${ }^{19}$. Estas funciones se unen a las reconocidas anteriormente en relación con el daño celular, el cáncer y el envejecimiento ${ }^{20-22}$.

Existen fundamentalmente 2 variedades de RL: las especies reactivas del oxígeno y las del nitrógeno. En las primeras encontramos el superóxido $\left(\mathrm{O}_{2}^{-}\right)$, el peróxido de hidrógeno $\left(\mathrm{H}_{2} \mathrm{O}_{2}\right)$ y el ión hidroxilo $\left(\mathrm{OH}^{-}\right)$. Una de las principales vías para la producción de $\mathrm{O}_{2}{ }^{-}$es a partir de la acción de la enzima NADPH oxidasa sobre el $\mathrm{O}_{2}$. Esta enzima se encuentra presente en múltiples células del organismo como neutrófilos ${ }^{23}$, fibroblastos ${ }^{24}$, células endoteliales ${ }^{25}$ y células mesangiales ${ }^{26}$. $\mathrm{El} \mathrm{H}_{2} \mathrm{O}_{2}$ deriva de la acción de la enzima superóxido dismutasa sobre el $\mathrm{O}_{2}^{-}$. Finalmente el ión $\mathrm{OH}^{-}$ se origina luego de la reducción del $\mathrm{H}_{2} \mathrm{O}_{2}$, apoyado por la actividad de moléculas de hierro ${ }^{19}$.

El segundo grupo de RL son las especies reactivas del nitrógeno, dentro de las que están el catión nitrosonium $\left(\mathrm{NO}^{+}\right)$, el anión nitroxilo $\left(\mathrm{NO}^{-}\right)$y el peroxinitrato $\left(\mathrm{ONOO}^{-}\right)$. La mayoría de estos productos tienen su origen en la interacción de moléculas de oxígeno con el óxido nítrico ${ }^{19}$.

En condiciones normales existe protección a los efectos tóxicos de estos RL por sustancias antioxidantes localizadas 
tanto intra como extracelulares. Sin embargo, cuando estos mecanismos de defensa fallan, las células pasan a un estado conocido como estrés oxidativo, desencadenando daños que pueden llegar a ser irreversibles e incluso provocar muerte celular $^{27,28}$.

Desde hace años se conoce que el estrés oxidativo desempeña un papel fundamental en la fisiopatología de la $\mathrm{HTA}^{29}$. Varias teorías han sido propuestas para explicar esta relación, pero las más estudiadas son la hemodinámica, que determina un aumento en la vasoconstricción arterial, y la estructural, que favorece el remodelado vascular. Los agentes reactivos del oxígeno pueden provocar un incremento en la vasoconstricción de los vasos sanguíneos sistémicos e intrarrenales ${ }^{30}$. Estas sustancias han mostrado capacidad para inactivar el oxido nítrico endotelial, lo que trae como consecuencia un incremento en la vasoconstricción ${ }^{31}$. Otra propuesta utilizada para explicar el aumento en la PA plantea que los agentes reactivos del oxígeno pueden incrementar la oxidación del ácido araquidónico, con la formación posterior de eicosanoides con propiedades vasoconstrictoras como la prostaglandina $\mathrm{F} 2 \alpha^{32}$ y la inhibición en la síntesis de $\mathrm{PGI}_{2}{ }^{33}$. Adicionalmente $\mathrm{el}_{2}{ }^{-}$ estimula la entrada de calcio en las células endoteliales ${ }^{34}$ y media la acción de otras sustancias vasoconstrictoras como la angiotensina II, tromboxano $A_{2}$, endotelina-1 y la norepinefrina ${ }^{35}$. Por otra parte, se sugiere que las especies del oxígeno tienen la capacidad de activar una serie de cascadas metabólicas en las células del músculo liso vascular que inducen remodelado de las arterias, lo que provoca rigidez de los vasos y estrechamiento de su luz ${ }^{36}$.

Clásicamente estas han sido las teorías utilizadas para explicar la influencia del estrés oxidativo en la fisiopatología de la HTA. Sin embargo, en los últimos años existen evidencias sobre el papel esencial del estrés oxidativo en la retención de sodio y agua en el tejido renal, provocando un incremento en las cifras de PA. La acumulación de RL en el intersticio renal ha demostrado que favorece la síntesis local de varias citocinas, las que estimulan la infiltración de células del sistema inmunológico. Estas, a su vez, incrementan la producción de hormonas prohipertensivas que llevan al desarrollo de $\mathrm{HTA}^{37}$.

\section{Inflamación renal e hipertensión sensible a sal}

La infiltración renal por células del sistema inmune y su relación con el desarrollo de HTA ha sido ampliamente estudiada. Kurokawa ${ }^{38}$ y Rodríguez-Iturbe et al. ${ }^{39}$ descubrieron, en modelos de animales con HSS, una infiltración significativa de células inmunes en diferentes secciones del tejido renal, asociada con altas concentraciones de angiotensina II. Entre los años 2005 y 2009 un grupo de investigadores demostraron por separado que los linfocitos tienen un sistema renina-angiotensina funcional ${ }^{40}$. En estos estudios se comprueba la expresión en las células T de angiotensinógeno, enzima convertidora de angiotensina, renina, además de ser capaces de producir niveles fisiológicos de angiotensina $\|^{41}$. Esto permite explicar por qué la presencia de células inmunes a nivel renal favorece la retención de sodio y agua y secundariamente HTA. Tal es el caso de modelos de HSS, donde la severidad de la HTA se correlaciona con un incremento en los niveles de angiotensina ॥ intrarrenal $\mathrm{e}$ infiltración de células inmunes ${ }^{42}$. En sendas investigaciones de Miguel et al. ${ }^{43,44}$ muestran que las células T favorecen el desarrollo de HSS y daño renal en ratas de laboratorio que presentan HSS. En la segunda de estas encuentran que, unido al incremento en la infiltración de linfocitos $T$ en el tejido renal de estos animales, la administración de un agente inmunosupresor como el tacrolimus disminuye la mencionada infiltración, reduce el estado oxidativo y la expresión renal de la enzima NADPH oxidasa. Esto provoca un descenso de la PA y de los niveles de albuminuria en los animales estudiados, reafirmando la relación entre inflamación renal e HSS. Además establece la posibilidad de reducir los niveles de PA con el uso de fármacos capaces de disminuir la inflamación y/o la infiltración de células inmunes en el tejido renal.

Otros estudios corroboran estas observaciones, pero además abordan la influencia de la angiotensina II formada en el intersticio renal y su posible papel en la inflamación renal $^{45}$. La angiotensina ॥ del intersticio renal desempeña una función importante en la regulación de la microcirculación renal, la función tubular y en la patogénesis de diversos daños tubulointersticiales ${ }^{46}$. También se han encontrado niveles superiores de angiotensina ॥ en el fluido del intersticio renal que en el plasma sanguíneo ${ }^{47,48}$, y se ha comprobado que los niveles de angiotensina ॥ en los vasos linfáticos renales no aumentan con la administración de esta sustancia en la circulación sanguínea de la arteria renal, por lo que es posible que la misma se forme intracelularmente y luego se mueva hacia el líquido intersticial ${ }^{49}$. A partir de la importancia de la angiotensina II en la fisiopatología de la HTA, es necesario ampliar las investigaciones sobre la formación de esta sustancia en el tejido renal, su interacción con otros elementos que participan en la inflamación renal, y la posible influencia para el desarrollo de HSS.

Los macrófagos son otras células del sistema inmune que han sido relacionadas con las cifras de PA. El vínculo entre la inflamación renal, el desarrollo de HSS y los trabajos que han probado la efectividad del uso de medicamentos inmunosupresores en el control de la PA, sugieren que los macrófagos desempeñan un rol fundamental en la aparición de HSS. Igualmente existen investigaciones demostrando la continua infiltración y participación de estas células y los linfocitos $T$ en el proceso de inflamación del intersticio renal ${ }^{38}$, que desencadena un aumento en la síntesis de angiotensina ॥ y posteriormente un incremento en los valores de la PA. Sin embargo, hay estudios que desaprueban esta teoría, pues establecen que los macrófagos participan en un mecanismo de defensa adaptativa que minimiza los efectos hipertensivos de la retención de sodio ${ }^{50}$. Apoyando esta idea, trabajos actuales indican que la depleción de los macrófagos favorece el desarrollo de HSS, debido a que estos participan en un mecanismo extrarrenal regulador del sodio intersticial ${ }^{51}$. Aún se necesitan nuevos estudios para conocer cuál es la verdadera función de estas células en el desarrollo de la HSS. Hasta el presente se conoce que formando parte del proceso inflamatorio del tejido renal están asociadas al incremento en las cifras de PA, mientras que a través de su actividad reguladora del sodio intersticial favorecen la reducción de las cifras tensionales.

Existe en la actualidad una teoría expuesta por Jonhson et al. ${ }^{12,52}$ que plantea la posibilidad de un proceso gradual y continuo en el desarrollo de HSS. Estos autores establecen 
que la PA sistémica elevada de manera continua desencadena daños renales con la formación de arteriolopatía aferente y enfermedad tubulointersticial, estimulando la producción de RL e incrementando la actividad de moléculas de adhesión para leucocitos, con la subsiguiente infiltración de linfocitos $\mathrm{T}$ y la activación del sistema reninaangiotensina-aldosterona. La acción prolongada de estas sustancias lleva a un estado de vasoconstricción en las arteriolas preglomerulares que reduce el filtrado glomerular y por tanto la excreción de sodio y agua, lo que favorece la aparición de HSS. Esta hipótesis permite esclarecer por qué existen distintos grados de sensibilidad a la sal en diferentes grupos de pacientes, pues esto depende de la intensidad de la afectación tubulointersticial de cada individuo en relación con el tiempo de evolución de la HTA. Además reafirma el papel de la inflamación renal en la aparición de HSS, y deja comprender que el daño provocado por la acción prolongada de altas cifras de PA en el tejido renal puede ser en parte el elemento necesario para estimular los procesos inflamatorios a este nivel que posteriormente llevarán a la HSS. La predisposición individual a sufrir afectación del intersticio renal a través de los mecanismos anteriores puede estar determinada por factores genéticos y ambientales que explicarían la complejidad de este tema cuando se estudian diversas poblaciones. Existen estudios demostrando que la angiotensina $\|$ producida a partir de la actividad de los linfocitos $\mathrm{T}$ posee propiedades proinflamatorias debido a que estimula la liberación de interleucina-1, interleucina6 y factor de necrosis tumoral $\alpha$, tanto a nivel local como sistémico ${ }^{53,54}$. Esta secuencia permite plantear la posibilidad de un círculo vicioso (fig. 1) que explica por qué estudios publicados al respecto muestran una prolongación de la inflamación del tejido renal en pacientes hipertensos, incrementando la resistencia de estos a los tratamientos antihipertensivos, y el riesgo de complicaciones futuras ${ }^{55}$.

\section{Relación entre inflamación renal, hipertensión sensible} a sal y trastornos autoinmunes

Las enfermedades autoinmunes se caracterizan por aumento en la actividad del sistema inmunológico. Los mecanismos propuestos para explicar el desarrollo de HTA en estas condiciones patológicas son múltiples ${ }^{56}$, por lo que nuestra exposición quedará reducida a aquellos que vinculan a estas entidades con la inflamación renal y el desarrollo de HSS. Kristensen y Andersen ${ }^{57}$ hallaron un incremento en anticuerpos séricos y reacciones de hipersensibilidad tardía contra antígenos vasculares en un grupo de pacientes hipertensos, demostrando un aumento en la reactividad autoinmune en estas personas. Por otra parte, se observa la presencia de efectos antihipertensivos con la depleción de linfocitos ${ }^{58}$, timectomía neonatal ${ }^{59}$, uso de sueros antitímicos ${ }^{58}$ y terapia con ciclofosfamida ${ }^{60}$ en varios estudios con modelos experimentales de HTA. Rodriguez-Iturbe et al. ${ }^{61}$ publicaron un artículo en el cual plantean que una actividad autoinmune a las proteínas de choque térmico (son chaperones moleculares que estimulan la reactividad autoinmune ${ }^{62}$ ) expresadas en los riñones puede ser un mecanismo que facilita un bajo grado de inflamación tubulointersticial a nivel renal por largos períodos de tiempo y que persiste, a pesar del uso de fármacos capaces de disminuir esta condición. Las proteínas de choque térmico se invocan en la continuidad del proceso

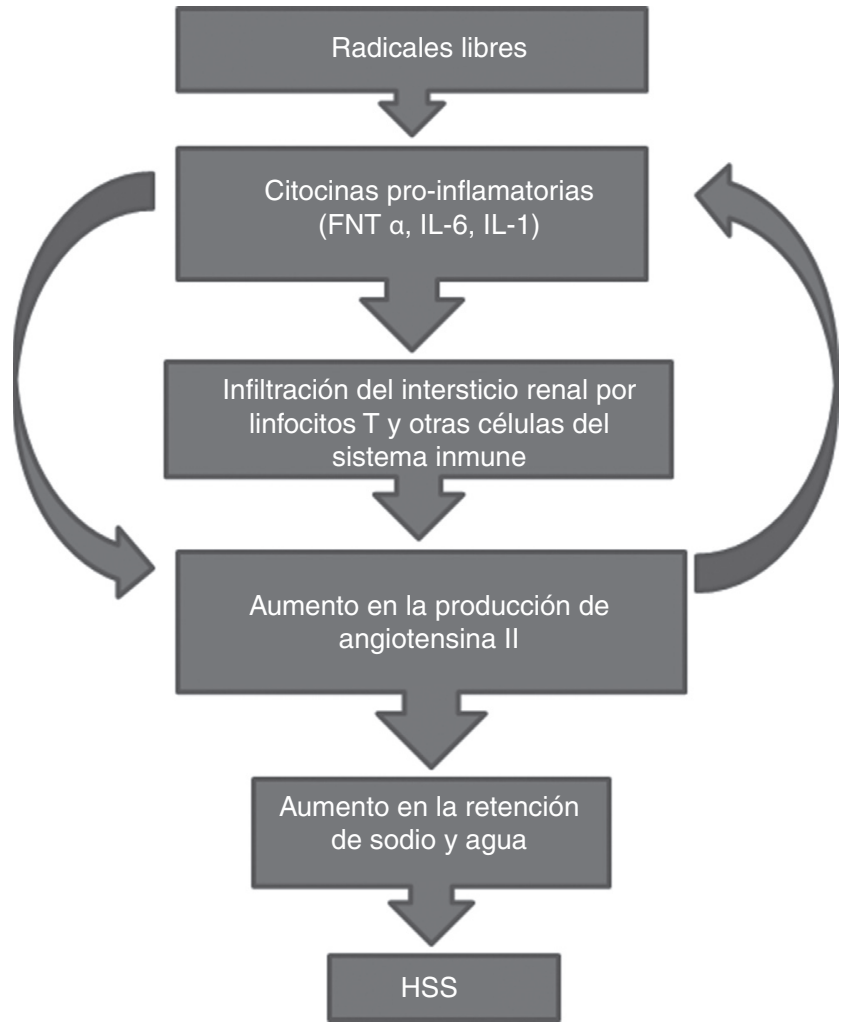

Figura 1 Mecanismos que explican la relación entre inflamación renal y el desarrollo de HSS. La figura muestra cómo la formación de RL inicia la producción de citocinas proinflamatorias que favorecen la infiltración renal de linfocitos $\mathrm{T}$ y otras células del sistema inmunológico, como los macrófagos. Este proceso estimula la síntesis y liberación de angiotensina II a este nivel, que incrementa la retención de sodio y agua y lleva finalmente a la HSS. Las flechas indican cómo el proceso se perpetúa, pues la inflamación renal incrementa los niveles de angiotensina ॥, y esta a su vez acrecienta la producción de citocinas que aumentan el proceso inflamatorio, desencadenando un ciclo que explica la continuidad de la inflamación renal observada en estos pacientes. FNT $\alpha$ : factor de necrosis tumoral $\alpha$; HSS: hipertensión sensible a sal, IL-1: interleucina-1; IL-6: Interleucina-6.

inflamatorio en los riñones, e incluso, al parecer facilitan su aparición a partir de un mecanismo autoinmine ${ }^{63}$. Esta inflamación incrementa la producción de angiotensina ॥ en esta zona, aumentando secundariamente los valores de PA. Varios investigadores han encontrado una sobreexpresión de este grupo de proteínas en los riñones de modelos de $\mathrm{HSS}^{64,65}$. Igualmente se ha logrado observar un aumento de anticuerpos sanguíneos contra las proteínas de choque térmico 70 y 65 en pacientes hipertensos, reaccionando con sus respectivos antígenos en el tejido renal ${ }^{66}$; esto favorece la aparición de procesos inflamatorios que llevan a la HSS como se ha explicado. El estudio de estas proteínas puede abrir un nuevo horizonte en la comprensión de la fisiopatología de algunas enfermedades autoinmunes y específicamente su relación con la HTA, pues parece evidente que una actividad autoinmune incrementada favorece su lesión, lo que aumenta la probabilidad de inflamación del intersticio renal. 
Tabla 1 Estudios que demuestran la efectividad de la terapia antioxidante en la reducción de las cifras de PA

\begin{tabular}{|c|c|c|}
\hline Referencia & Muestra & Principales resultados \\
\hline Chen et al. ${ }^{69}$ & $\begin{array}{l}\text { 15,317 personas pertenecientes al Third } \\
\text { National Health Examination Survey }\end{array}$ & $\begin{array}{l}\text { Los } \alpha \text {-carotenos, } \beta \text {-carotenos y la vitamina C } \\
\text { se encuentran inversamente relacionados } \\
\text { con las cifras de PA }\end{array}$ \\
\hline Appel et al. ${ }^{70}$ & $\begin{array}{l}459 \text { adultos, divididos en grupo control, grupo } \\
\text { con dietas ricas en frutas y vegetales, y grupo } \\
\text { con dietas ricas en frutas y vegetales y bajo } \\
\text { consumo de grasas saturadas }\end{array}$ & $\begin{array}{l}\text { Los pacientes que recibieron dietas ricas en } \\
\text { frutas y vegetales presentan una reducción } \\
\text { significativa de la PA comparado con el grupo } \\
\text { control. Esta reducción es mayor entre los } \\
\text { pacientes hipertensos }\end{array}$ \\
\hline Rodrigo et al. ${ }^{71}$ & $\begin{array}{l}110 \text { pacientes con HTA esencial divididos en } \\
\text { grupo control y grupo de estudio. Seguimiento } \\
\text { durante } 8 \text { semanas }\end{array}$ & $\begin{array}{l}\text { La administración de vitaminas C y E reduce } \\
\text { las cifras de PA sistólica y diastólica. Además } \\
\text { aumenta la capacidad antioxidante de los } \\
\text { eritrocitos y del plasma sanguíneo }\end{array}$ \\
\hline Rodrigo et al. ${ }^{72}$ & $\begin{array}{l}66 \text { pacientes divididos en grupo estudio con } \\
31 \text { pacientes hipertensos y grupo control } \\
\text { con } 35 \text { pacientes normotensos }\end{array}$ & $\begin{array}{l}\text { Los niveles plasmáticos de vitamina } \mathrm{C} \text { se } \\
\text { correlacionan negativamente con las cifras } \\
\text { de PA sistólica y diastólica }\end{array}$ \\
\hline
\end{tabular}

Apoyando los anteriores estudios, un grupo de investigadores demuestran que existe una reducción en las cifras de PA y en la excreción renal de citocinas en pacientes hipertensos con psoriasis y artritis reumatoide que han sido tratados con mofetil micofenolato ${ }^{67}$. Este medicamento es un inmunosupresor y su mecanismo de acción en la reducción de la PA puede deberse a una disminución en la inflamación del intersticio renal. La administración de lisinopril (inhibidor de la enzima convertidora de la angiotensina) e ICI D8731 (bloqueador de los receptores de angiotensina ॥) en ratones con HTA espontánea, demuestra la capacidad de estos para lograr una normalización de la PA, y una reducción significativa en la infiltración de células T en el tejido renal de estos animales comparado con controles ${ }^{68}$.

\section{Terapia antioxidante y perspectivas futuras}

A partir del conocimiento sobre la relación entre estrés oxidativo e HTA, varios estudios con agentes antioxidantes han sido diseñados para lograr una reducción en las cifras de PA, alcanzándose resultados positivos ${ }^{69-72}$ (tabla 1).

Sin embargo, existen otros autores que no han encontrado beneficios con la administración de antioxidantes en la terapéutica de la HTA ${ }^{73,74}$. Hay aspectos que deben tenerse en cuenta, y que no han sido correctamente evaluados en todos los estudios sobre el uso de antioxidantes en este escenario, lo que explica la existencia de resultados tan diversos. Un primer punto debe ser la posibilidad de una dosis insuficiente de los distintos fármacos antioxidantes utilizados en la práctica clínica, o que de alguna manera no exista una adecuada correlación entre la posología de los medicamentos antioxidantes y la concentración de los RL en el organismo, pues existen factores que pueden estimular el desarrollo de estrés oxidativo como el aumento de metales pesados, xenobióticos, el hábito de fumar, la adriamicina, radiaciones ultravioletas, traumatismos y el ejercicio físico extenuante ${ }^{75}$. Estos factores deben ser modificados debido a que su persistencia puede interferir en la eficacia del tratamiento antioxidante. Además, es necesario diferenciar los niveles de RL hallados a nivel sistémico y los presentes en el tejido renal. Estos últimos son los que están directamente involucrados en el proceso de inflamación renal $y$, por tanto, en la retención de sodio y el desarrollo de HSS.

En el futuro deben continuar desarrollándose nuevos medicamentos capaces de actuar sobre los mecanismos del estrés oxidativo y/o de la inflamación renal. Esto permitiría ampliar nuestro arsenal terapéutico para lograr un tratamiento más integral de los pacientes con HTA, y específicamente, aquellos que padecen HSS.

\section{Conclusiones}

Existe una relación demostrada entre el estrés oxidativo, inflamación del intersticio renal y el desarrollo de HSS. La infiltración de linfocitos $\mathrm{T}$ y otras células del sistema inmunológico en el tejido renal provoca un aumento en los niveles de angiotensina ॥, que deriva en un incremento en la retención de sodio y agua, favoreciendo la aparición de HSS. La relación entre HTA y enfermedades autoinmunes puede explicarse en parte sobre la base de estos conocimientos. El uso adecuado de medicamentos antioxidantes y el desarrollo de nuevos fármacos en este campo pueden incrementar las opciones terapéuticas de los pacientes afectados.

\section{Conflicto de intereses}

Los autores declaran no tener ningún conflicto de intereses.

\section{Bibliografía}

1. Staessen JA, Wang J, Bianchi G, et al. Essential hypertension. Lancet. 2003;361:1629-41.

2. Lawes CM, Vander Hoorn S, Rodgers A. Global burden of bloodpressure-related disease, 2001. Lancet. 2008;371:1513-8.

3. Mancia G, Fagard R, Narkiewicz K, et al. 2013 ESH/ESC guidelines for the management of arterial hypertension. J Hypertens. 2013;31:1281-357.

4. Ardiles L, Mezzano S. Rol del riñón en la hipertensión salsensible. Rev Med Chile. 2010;138:862-7. 
5. Hall JE, Granger JP, do Carmo JM, et al. Hypertension: Physiology and pathophysiology. Compr Physiol. 2012;2:2393-442.

6. Elliot P, Stamler J, Nichols R, et al. Intersalt revisited: further analysis of 24 hour sodium excretion in blood pressure within and across populations. BMJ. 1996;312:1249-53.

7. Guild SJ, McBryde FD, Malpas SC, et al. High dietary salt and angiotensin II chronically increase renal sympathetic nerve activity: A direct telemetric study. Hypertension. 2012;59:614-20.

8. Weinberg M, Fineberg N. Sodium and volume sensitivity of blood pressure. Age and pressure change over time. Hypertension. 1991;18:67-71.

9. Franco M, Sánchez-Lozada LG, Bautista R, et al. Pathophysiology of salt-sensitive hypertension: A new scope of an old problem. Blood Purif. 2008;26:45-8.

10. Herrera Acosta J. Hipertensión arterial dependiente de sal. Arch Cardiol Mex. 2001;71 Supl 1:S76-80.

11. González Toledo R, Franco M. ¿Es la hipertensión sensible a sal una enfermedad inflamatoria? Papel de los linfocitos y macrófagos. Arch Cardiol Mex. 2012;82:312-9.

12. Johnson RJ, Herrera JA, Schreiner G, et al. Subtle acquired renal injury as a mechanism of salt-sensitive hypertension. $N$ Engl J Med. 2002;346:913-23.

13. Johnson AG, Nguyen TV, Davis D. Blood pressure is linked to salt intake and modulated by the angiotensinogen gene in normotensive and hypertensive elderly subjects. J Hypertens. 2001;19:1053-60.

14. Poch E, González D, Giner V, et al. Molecular basis of salt sensitivity in human hypertension. Evaluation of reninangiotensin-aldosterone system gene polymorphisms. Hypertension. 2001;38:1204-9.

15. Shimoike H, Iwai N, Kinoshita M. Genetic analysis of renin gene expression in the central nervous system of spontaneously hypertensive rats. Neurosci Lett. 1997;221:133-6.

16. Pamies-Andreu E, Ramirez-Lorca R, Stiefel García-Junco P, et al. Renin-angiotensin-aldosterone system and G-protein beta-3 subunit gene polymorphisms in salt sensitive essential hypertension. J Hum Hypertens. 2003;17:187-91.

17. Weinberger MH, Fineberg NS, Fineberg SE, et al. Salt sensitivity, pulse pressure and death in normal and hypertensive humans. Hypertension. 2001;37:429-32.

18. Morimoto A, Uzu T, Fujii T, et al. Sodium sensitivity and cardiovascular events in patients with essential hypertension. Lancet. 1997;350:1734-7.

19. Dröge W. Free radicals in the physiological control of cell function. Physiol Rev. 2002;82:47-95.

20. Harman D. Ageing: A theory based on free radical and radiation chemistry. J Gerontol. 1956;11:298-300.

21. Harman D. The ageing process. Proc Natl Acad Sci U S A. 1981;78:7124-8.

22. Harman D. La teoría gerontológica de los radicales libres. Rev Esp Geriatr Gerontol. 2001;36:187.

23. Griendling KK, Sorescu D, Ushio-Fukai M. NAD(P)H oxidase: Role in cardiovascular biology and disease. Circ Res. 2000;86:494-501.

24. Jones SA, Wood JD, Coffey MJ, et al. The functional expression of p47-phox and p67-phox may contribute to the generation of superoxide by NADPH oxidase-like system in human fibroblasts. FEBS Lett. 1994;355:178-82.

25. Jones SA, O'Donell VB, Wood JD, et al. Expression of phagocyte NADPH oxidase components in human endothelial cells. Am J Physiol Cell Physiol. 1996;271:C626-34.

26. Jones SA, Hancock J, Jones OTG, et al. The expression of NADPH oxidase components in human glomerular mesangial cells: Detection of protein and mRNA for p47phax, p67phax, and p22 phax. J Am Soc Nephrol. 1995;5:1483-91.

27. Céspedes Cabrera T, Sánchez Serrano D. Algunos aspectos sobre el estrés oxidativo, el estado antioxidante y la terapia de suplementación. Rev Cubana Cardiol. 2000;14:55-60.
28. Llacuna L, Mach N. Papel de los antioxidantes en la prevención del cáncer. Rev Esp Nutr Hum Diet. 2012;16:16-24.

29. Makino A, Skelton MM, Zou AP, et al. Increased renal medullary oxidative stress produces hypertension. Hypertension. 2002;39:667-72.

30. Clerk A, Fuller SJ, Michael A, et al. Stimulation of «stressactivated» mitogen-activated protein kinases/c-Jun N-terminal kinases, and p38 mitogen-activated protein kinases in perfuse rat hearts by oxidative and other stresses. J Biol Chem. 1998;273:7228-34.

31. Cai H, Harrison DG. Endothelial dysfunction in cardiovascular diseases: Role of oxidant stress. Circ Res. 2000;87:840-4.

32. Takahashi K, Nammour TM, Fukunaga M, et al. Glomerular actions of a free radical-generated novel prostaglandin, 8-epiprostaglandin F2 $\alpha$ Evidence for interaction with thromboxane A2 receptors. J Clin Invest. 1992;90:136-41.

33. Zou MH, Ulrich V. Peroxynitrite formed by simultaneous generation of nitric oxide and superoxide selectively inhibits bovine aortic prostacyclin synthase. FEBS Lett. 1996;382:101-4.

34. Lounsbury KM, Hu Q, Ziegelstein RC. Calcium signaling and oxidant stress in the vasculature. Free Rad Biol Med. 2000;28:1362-9.

35. Schnackenberg CG. Physiological and pathophysiological roles of oxygen radicals in the renal microvasculature. Am J Physiol Regul Integr Comp Physiol. 2002;282:R335-42.

36. Kunsch C, Medford RM. Oxidative stress as a regulator of gene expression in the vasculature. Circ Res. 1999;85:753-66.

37. Rodriguez-Iturbe $B$, Quiroz $Y$, Nava M, et al. Reduction of renal immune cell infiltration results in blood pressure control in genetically hypertensive rats. Am J Physiol Renal Physiol. 2002;282:F191-201.

38. Kurokawa K. Kidney, salt and hypertension: How and why. Kidney Int. 1996;49 Suppl 55:S46-51.

39. Rodríguez-Iturbe B, Pons H, Quiroz Y, et al. Mycophenolate mofetil prevents salt-sensitive hypertension resulting from angiotensin II exposure. Kidney Int. 2001;59:2222-32.

40. Jurewicz M, McDermott DH, Sechler JL, et al. Human $T$ and natural killer cells posses a functional renin-angiotensin system: further mechanisms of angiotensin II-induced inflammation. J Am Soc Nephrol. 2007;18:1093-102.

41. Hotch NE, Guzik TJ, Chen W, et al. Regulation of T-cell function by endogenously produced angiotensin II. Am J Physiol Regul Integr Comp Physiol. 2009;296:R208-16.

42. Franco M, Martínez F, Quiroz Y, et al. Renal angiotensin II concentration and interstitial infiltration of immune cells are correlated with blood pressure levels in salt-sensitive hypertension. Am J Physiol Reg Integr Comp Physiol. 2007;293: R251-6.

43. De Miguel C, Das S, Lund H, et al. T lymphocytes mediate hypertension and kidney damage in Dahl salt-sensitive rats. Am J Physiol Regul Integr Comp Physiol. 2010;298:R1136-42.

44. De Miguel C, Guo C, Lund H, et al. Infiltrating T lymphocytes in the kidney increase oxidative stress and participate in the development of hypertension and renal disease. Am J Physiol Renal Physiol. 2011;300:F734-42.

45. Nishiyama A, Seth DM, Navar LG. Renal interstitial fluid angiotensin I and angiotensin I| concentrations during local angiotensin converting enzyme inhibition. J Am Soc Nephrol. 2002;13:2207-12.

46. Mitchell KD, Navar LG. Enhanced tubuloglomerular feedback during peritubular infusions of angiotensins I and II. Am J Physiol. 1988;255:F383-90.

47. Wilcox CS, Dzau VJ. Effect of captopril on the release of the components of the renin-angiotensin system into plasma and lymph. J Am Soc Nephrol. 1992;2:1241-50.

48. Nishiyama A, Seth DM, Navar LG. Renal interstitial fluid concentrations of angiotensins I and II in anesthetized rats. Hypertension. 2002;39:129-34. 
49. Bailie MD, Rector Jr FC, Seldin DW. Angiotensin II in arterial and renal venous plasma and renal lymph in the dog. J Clin Invest. 1971;50:119-26.

50. Machnik A, Dhalmann A, Kopp C, et al. Mononuclear phagocyte system depletion blocks interstitial tonicity-responsive enhyancer binding protein/vascular endothelial growth factor $\mathrm{C}$ expression and induces salt-sensitive hypertension in rats. Hypertension. 2010;55:755-61.

51. Titze J, Machnik A. Sodium sensing in the interstitium and relationship to hypertension. Curr Opin Nephrol Hypertens. 2010;19:385-92.

52. Johnson RJ, Schreiner JF. Hypothesis: 1. The role of acquired tubulointerstitial disease in the pathogenesis of salt-sensitive hypertension. Kidney Int. 1997;52:1169-79.

53. Brasier AR, Recinos III A, Eledrisi MS. Vascular inflammation and the renin-angiotensin system. Arterioscler Thromb Vasc Biol. 2002;22:1257-66.

54. Sekiguchi K, Li X, Coker M, et al. Cross-regulation between the renin-angiotensin system and inflammatory mediators in cardiac hypertrophy and failure. Cardiovasc Res. 2004;63:433-42.

55. Rodriguez-Iturbe B, Romero F, Johnson RJ. Pathophysiologic mechanisms of salt-dependent hypertension. Am J Kidney Dis. 2007; 50:655-72.

56. Ryan MJ. The pathophysiology of hypertension in systemic lupus erythematosus. Am J Physiol Regul Integr Comp Physiol. 2009;296:R1258-67.

57. Kristensen BO, Andersen PL. Autoantibodies in untreated and treated essential hypertension. Acta Med Scan. 1978;203:55-9.

58. Svendsen UG. Evidence for an initial, thymus independent and a chronic, thymus-dependent phase of DOCA and salt hypertension in mice. Acta Path Microbiol Scand A. 1976;84:523-8.

59. Bataillard P, Freiche JC, Vincent M, et al. Effects of neonatal thymectomy on blood pressure and immunological characteristics of the genetically hypertensive rats of the Lyon strain. J Hypertens. 1986;4 Suppl 3:S5455-67.

60. Khraibi AA, Norman Jr RA, Dzielak DJ. Chronic immunosuppression attenuates hypertension in Okamoto spontaneously hypertensive rats. Am J Physiol. 1984;247:H722-6.

61. Rodríguez-Iturbe B, Vaziri ND, Herrera-Acosta J, et al. Oxidative stress, renal infiltration of immune cells and salt-sensitive hypertension: All for one and one for all. Am J Physiol Renal Physiol. 2004;286:F606-16.

62. Kaufmann SHE. Heat shock proteins and the immune response. Immunol Today. 1990;11:129-36.
63. Pons H, Ferrebuz A, Quiroz Y, et al. Immune reactivity to heat shock protein 70 expressed in the kidney is cause of salt-sensitive hypertension. Am J Physiol Renal Physiol. 2013;304:F289-99.

64. Ishizaka N, Aizawa T, Ohno M, et al. Regulation and localization of HSP70 and HSP25 in the kidney of rats undergoing long-term administration of angiotensin II. Hypertension. 2002;39:122-8.

65. Bravo J, Quiroz Y, Pons H, et al. Vimentin and heat shock protein expression are induced in the kidney by angiotensin and by nitric oxide inhibition. Kidney Int. 2003;64 Suppl 86:S46-51.

66. Pockley AG, de Faire U, Kiessling R, et al. Circulating heat shock protein and heat shock protein antibody levels in established hypertension. J Hypertens. 2002;20:1815-20.

67. Herrera J, Ferrebuz A, MacGregor EG, et al. Mycophenolate mofetil improves hypertension in patients with psoriasis and rheumatoid arthritis. J Am Soc Nephrol. 2006;17 Suppl 3:S218-25.

68. Geiger H, Fierlbeck W, Mai M, et al. Effects of early and late antihypertensive treatment on extracellular matrix proteins and mononuclear cells in uninephrectomized SHR. Kidney Int. 1997;51:750-61.

69. Chen J, He J, Hamm L, et al. Serum antioxidant vitamins and blood pressure in the United States population. Hypertension. 2002;40:810-6.

70. Appel LJ, Moore TJ, Obarzanek E, et al. A clinical trial of the effects of dietary patterns on blood pressure. DASH Collaborative Research Group. N Engl J Med. 1997;336:1117-24.

71. Rodrigo R, Prat H, Passalacqua J, et al. Decrease in oxidative stress through supplementation of vitamins $C$ and $E$ is associated with a reduction in blood pressure in patients with essential hypertension. Clin Sci (Lond). 2008;114:625-34.

72. Rodrigo R, Prat H, Passalacqua J, et al. Relationship between oxidative stress and essential hypertension. Hypertens Res. 2007;30:1159-67.

73. Kim MK, Sasaki S, Sasazuki S, et al. Lack of long-term effect of vitamin C supplementation on blood pressure. Hypertension. 2002;40:797-803.

74. Heart Protection Study Collaborative Group. MRC/BHF heart protection study of antioxidant vitamin supplementation in 20,536 high-risk individuals: A randomized placebo-controlled trial. Lancet. 2002;360:23-33.

75. Gutiérrez Maydata A. Radicales libres derivados del oxígeno y estrés oxidativo. En: Caballero López A, editor. Terapia intensiva., 1, 2. ${ }^{a}$ ed. La Habana: Ciencias Médicas; 2006. p. 169-74. 